\title{
An accurate target tracking method in wireless sensor networks
}

\author{
Hanen Ahmadi ${ }^{1,2,3}$, Ridha Bouallegue ${ }^{2}$ \\ ${ }^{1}$ Mediterranean Institute of Technology, South Mediterranean University, Tunis, Tunisia \\ ${ }^{2}$ Innov'COM, Supcom, University of Carthage/University of Tunis El Manar, Tunis, Tunisia \\ 32ELEDIA Research Center, ELEDIA@Innov'COM-University of Carthage, Tunis, Tunisia
}

\begin{tabular}{l}
\hline Article Info \\
\hline Article history: \\
Received Aug 19, 2021 \\
Revised Dec 13, 2021 \\
Accepted Jan 10, 2022 \\
\hline
\end{tabular}

Keywords:

Machine learning

RSSI

Wireless sensor networks

Wireless tracking

\begin{abstract}
Localization is a challenging research issue in various sectors of activity, particularly in dynamic indoor environment with high perturbation. Many existing localization techniques in wireless sensor networks are not efficient because of many constraints such as the high cost, the memory and energy limitation and the environmental noise effects. Thus, the development of novel methods of localization has become a great concern for the wireless sensor networks. In this paper, we propose a tracking method that combines regression tree and Kalman smoother filtering. Previously, regression tree has been suggested for static positioning by means of received signal strength indicator measurements. In this work, we employ this strategy to solve the mapping relation between these measurements and the target position by means of an optimized propagation model. Moreover, the predicted position considered as the observed information is introduced to the Kalman smoother algorithm, to have more precise state of the moving target. The proposed algorithm has been assessed and compared to other existing methods using real measurements of the received power by the moving target in an indoor environment. The evaluation shows that our solution outperforms other methods regarding localization accuracy.
\end{abstract}

This is an open access article under the CC BY-SA license.

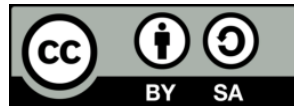

\section{Corresponding Author:}

Hanen Ahmadi

Mediterranean Institute of Technology, South Mediterranean University

Tunis, Tunisia

Email: hanen.ahmadi@medtech.tn

\section{INTRODUCTION}

Wireless sensor networks (WSNs) have gained great importance in the last few years in various monitoring and control applications such as military applications, smart building, health monitoring [1], recognition, environment control and precision farming [2]. This interest is expected to grow further with the high evolution of wireless systems. An increasing number of applications have been related to distributed monitoring by exploiting real-time position estimation.

Localization [3], [4] has became a very important need in these applications. Accordingly, researchers continue seeking efficient solutions for localization and tracking services which should be able to make a tradeoff between robustness, precision and real time availability. Global positioning system (GPS) is popular method of the localization service; however, its employment in indoor environments is not possible. Therefore, researchers seek for alternative solutions based on WSNs. In fact, many strategies using different metrics like received signal strength indicator (RSSI) [5]-[7], angle of arrival (AOA) [8], time of arrival (TOA) [9] and impulse responses (IR) [10], have been suggested. Localization using RSSI has been proposed as this radio indicator is always available and easily measured, while the other existing techniques often require expensive and complex hardware and present many constraints such as the high cost and the memory and energy limitation. On the other side, indoor environments present many constraints like interference, 
reflections, shadowing due to obstacles and pathloss fading. Consequently, RSSI is submitted to high level of fluctuations that will cause negative effects on the accuracy of the localization results. Particularly, the main challenge is to reduce this perturbation in dynamic scenario. So, it has been suggested to develop learning by example (LBE) solutions which proved their suitability in the understanding of the complex relation between the RSSI behavior and the target position. Many researches propose Machine learning based methods for localization systems such as support vector machines (SVMs) [11], neural networks (NNs), and Fuzzy Logic [12]. Accordingly, with machine learning methods, it is simpler to model the complex and dynamic behavior of RSSI in WSNs. Also, predicting mobile positions solutions using filtering have been increasingly employed [13]-[15].

The contributions of this paper are listed as follows: First contribution is emphasized on the framework of building the fingerprint database by means of an adequate propagation model applied to the multiple distributed training samples in order to address RSSI fluctuations problem. Second contribution proposes a tracking strategy of a moving node that combines regression tree and Kalman Smoother to have better performance. It uses a decision tree as a predictive model which maps the observations of training RSSI to the training positions. The estimated location by means of the RT is introduced to the Kalman smoother algorithm as the observation information, to provide more accurate state of the target. The proposed algorithm has been experimentally assessed using real measurement of a mobile target in an office room. The first section introduces the problematic. Then, Sect. 2 is devoted to explain the proposed method. In this section, the RT based localization algorithm using RSSI parameters is presented. Finally, the last section is devoted to analyze the experimental results.

The development of smart communication technologies has enabled the emergence of new Location based services (LBSs) applications [16]-[18]. Indeed, indoor localization is a basic process in robotics field [19], [20]. Simultaneous localization and mapping (SLAM) is proposed [21], enabling localization and building maps of an unknown environment. The authors combine SLAM with extended Kalman filter (EKF) to overcome the problem of having noisy acquired data. The simulation validation shows accurate and consistent positioning particularly when the landmarks number is increased. However, in the context of mobile autonomous robots which are limited in terms of energy, the SLAM method requires higher computational cost and its complexity should be studied specially when dealing with large maps.

Geolocalization is well used to develop efficient routing algorithm [22]. Developing routing method for emergency healthcare applications is a challenging topic. Singh et al. [23] proposed an efficient routing algorithm based on geolocation (W-GeoR) for VANET's health monitoring applications. The W-GeoR method is emphasized on selecting the best next-hop node to determine the optimal path.

The advancement of micro-electro-mechanical systems (MEMS) has enabled the growth of the unmanned aerial vehicle (UAV). Mostafa et al. [24], design an agent-based autonomous flight control (AFC) architecture for UAV, enabling autonomous navigation and routing. Among the proposed functionalities, localization is suggested to perform the tracking of UAV. The evaluation results confirm the performance of the proposed architecture in terms of precision, quality and power consumption. In similar context, autonomous vehicles have seen tremendous growth in recent years [25]. Localization and motion detection are basic parts of autonomous vehicle. Several recent studies [26]-[28] have suggested solutions to determine the path tracking of the autonomous vehicle. Vivacqua et al. [29] have proposed a low-cost architecture in terms of required equipments and algorithm complexity. It uses filtering method to reduce data uncertainty and improve the solution robustness.

Many researchers have studied range-based localization using specific metric to enhance localization accuracy. For instance, Thimmaiah and Mahadevan [30] have proposed a TOA based localization method in wireless sensor networks. It has been proved that this method outperforms other existing approaches in terms of localization error tolerance. While ToA based localization systems provide accurate results, it is essential to have synchronized clocks. Synchronization has a negative impact on the localization system complexity.

\section{RESEARCH METHOD}

\subsection{Problem formulation}

In this section, we descibe the experimental environment where the performance of the localization algorithm will be evaluated. We consider the indoor environment with size $X=9[\mathrm{~m}]$ and $Y=7.1[\mathrm{~m}]$. As shown in Figure 1. A set of $\mathrm{L}$ reference nodes (anchors) are available at fixed positions. As we consider the dynamic scenario, the moving target will acquire the RSSI data from anchors. The collected RSSI data will be provided at particular positions. The IEEE802.15.4 standard working at the operating frequency $f=2.4$ $[\mathrm{GHz}]$ has been used in this experiment. 


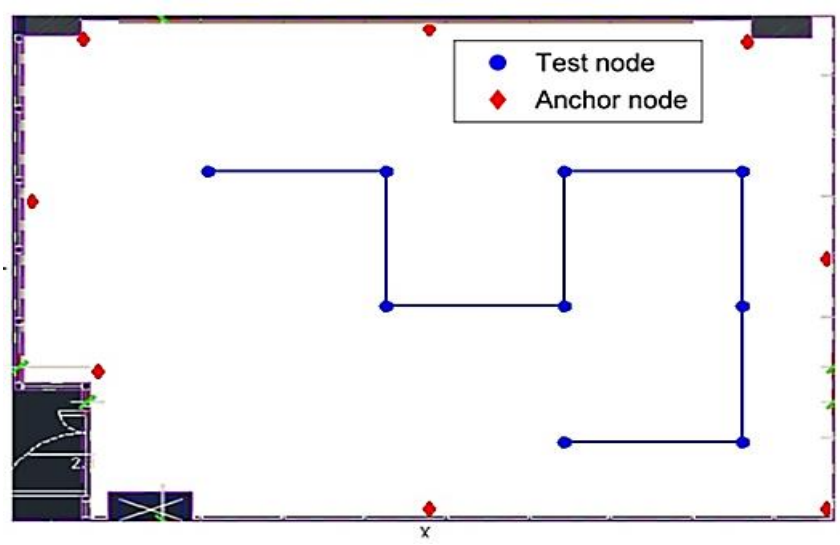

Figure 1. Indoor environment

The position state evolution over time is depicted in Figure 2. As shown, each current position estimation $X_{t+1}$ is based on the previous estimation $X_{t}$ by predicting each time the covariance error denoted $P$. The updating equations yield to more refined position estimation $Y$ at each time instant.

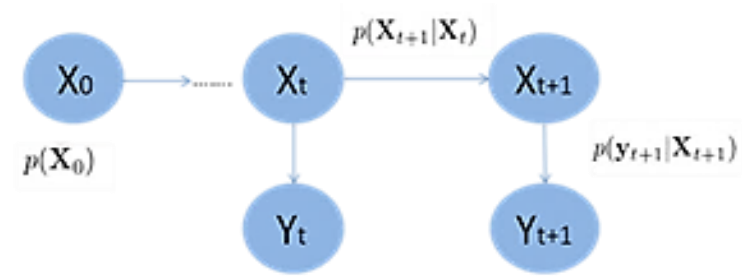

Figure 2. Target tracking problem

We denote unknown state vector at time $\mathrm{t}$ as $\mathrm{x}(\mathrm{t})$, including the position of the moving sensor. The adopted mobility [31] equation is given by (1):

$$
x(t)=F x(t-1)+W
$$

with $\mathrm{F}$ is the state transition matrix and $W=N(0, Q)$ is the the noise term given by a Gaussian distribution with zero mean and covariance $Q$. The observation equation including the measurement is given by (2):

$$
z(t)=H x(t)+V
$$

with $\mathrm{H}$ is the observation matrix and $V=N(0, R)$ is the observation noise with covariance denoted $R$.

\subsection{Proposed tracking algorithm}

\subsubsection{Channel modeling}

To characterize the indoor environment propagation, it has been suggested to refer to the pathloss log-normal model [32] for its efficiency and simple implementation. Accordingly, the received power can be defined as (3),

$$
P_{r x}=P_{t x}+K_{c}-10 \eta \log \left(\frac{d}{d_{0}}\right)+X_{\sigma}
$$

where $P_{t x}$ is the transmitted power, $\eta$ is the pathloss coefficient which depends on the environment characteristic, $d_{0}$ is the reference distance, $d$ is the transmitter-receiver distance and $K_{c}$ is a propagation constant given by (4).

$$
K_{c}=20 \log \left(\frac{4 \pi}{\lambda}\right)
$$


The noise $X_{\sigma}$ is modeled by a Gaussian random variable with zero-mean and variance $\sigma$. Let us consider an optimized channel modeling by selecting an optimal pathloss exponent $\eta$ by focusing on minimizing the minimum mean square error (MMSE) given by (5):

$$
\min _{\eta}\left[\left|P_{r x}(\eta)-R S S I_{r x}\right|^{2}\right]
$$

where $R_{S S I}$ is the RSSI measured by one of the selected anchors. As discussed in [17], the optimized channel model is emphasized on the changing behavior of the pathloss exponent according to the distance. Thus, the new model focuses on two different pathloss exponents $\eta_{1}$ and $\eta_{2}$ as described in the following equation.

$$
P_{r x}=\left\{\begin{array}{l}
P_{t x}+K_{c}-10 \eta_{1} \log \left(\frac{d}{d_{0}}\right) \text { ifd }<20 \lambda \\
P_{t x}+K_{c}-10 \eta_{2} \log \left(\frac{d}{d_{0}}\right) \text { otherwise }
\end{array}\right.
$$

\subsubsection{Observation model: RT-based localization}

This section aims at introducing the RT based algorithm using RSSI [33]. The algorithm starts with defining M training samples presented in uniform distribution as indicated in Figure 3, where $\delta$ is the interval between two samples. As shown in Figure 4, the RSSI transmitted by the anchor nodes and collected by the moving target represent the new input data to RT method. The predicted result will be considered as the observed data $z(t)$. The proposed model is presented in the following sub-section.

a) Definition of the training data: Machine learning methods are emphasized on two main steps, the training step and the testing or prediction step. The input information $P=\left(\left\{P_{r x, m}^{l}\right\}\right)_{m=1}^{M}$, is the received signal strength at samples $m=1, \ldots, M$ and transmitted by reference node $l$. The location of each training sample is denoted as $r_{m}=\left(x_{m}, y_{m}\right)$.

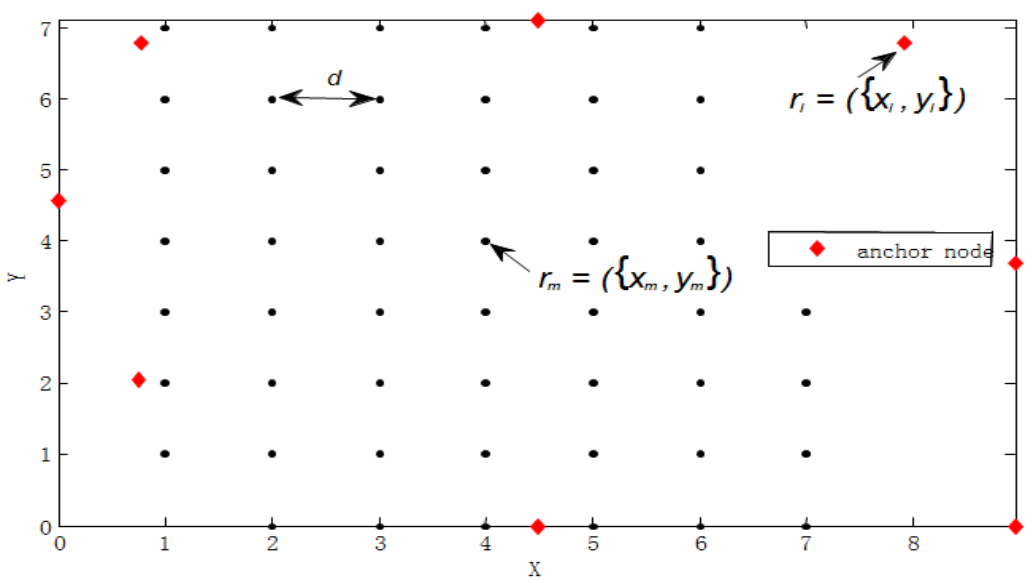

Figure 3. Training data

b) Regression tree algorithm using RSSI: This section describes the RT method. As presented in Figure 4. As shown in Figure 4, the algorithm starts by the training phase, where the defined training samples with their corresponding locations $\underline{r}_{m}=\left(\left\{x_{m}, y_{m}\right\}_{m=1}^{M}\right)$ are the input training data. The main goal of the training process is to construct the model which maps the RSSI data to the locations. This method is based on minimizing the mean square error $\varepsilon$ given by the following expression where $r_{m}$ is the actual training position and $\tilde{r}$ is the estimated position during the training.

$$
\begin{aligned}
& \varepsilon_{t}=\frac{1}{M_{l}} \sum_{m=1}^{M_{l}}\left(r_{m}-\tilde{r}\right)^{2} \\
& \tilde{r}=\frac{1}{M} \sum_{m=1}^{M} \underline{r}_{m}
\end{aligned}
$$

Then, the algorithm focuses on splitting the training set and minimizing the error resulting from the each 
split $\varepsilon^{(s p l i t)}$.

$$
\varepsilon^{(s p l i t)}=\frac{M_{l}^{(l e f t)}}{M_{l}} \sum_{m: P_{m}^{i d}}\left(\tilde{r}_{1}-r_{m}\right)+\frac{M_{l}^{(\text {right })}}{M_{l}} \sum_{m: P_{m}^{i d}}\left(\tilde{r}_{2}-r_{m}\right)
$$

$M_{l}^{(r i g h t)}$ is the number of cases in the right subset, $\tilde{r}_{1}$ and $\tilde{r}_{2}$ are the predicted positions presenting the two sub-nodes left and right resulting from the split.

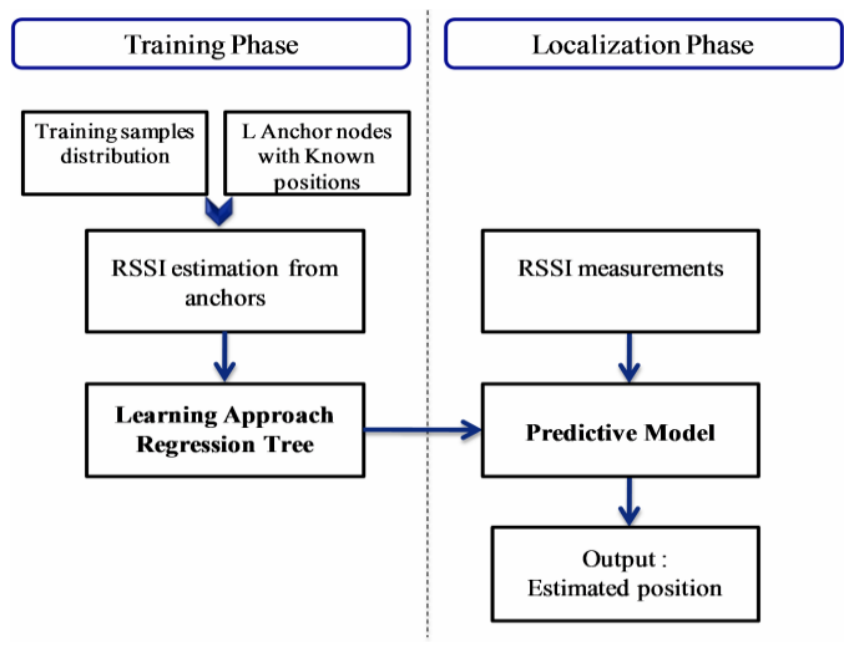

Figure 4. RT-based localization method

c) Kalman smoother: As previously mentioned, the $\mathrm{KS}$ is introduced to have more refined position estimation. One of the common methods used in KS is the Rauch-Tung-Striebel (RTS) smoother algorithm. RTS is an efficient two-pass algorithm operating in two stages: the forward and the backward steps. The first one is defined as the regular Kalman filter (KF) using recursive model for state estimation. The different steps of the KS method are illustrated in Figure 5. The goal of prediction phase of KS is to estimate the future position based on the current location as given by (10).

$$
x(t / t-1)=F x(t-1)+W
$$

The covariance is given by (11):

$$
P(t / t-1)=F P(t-1) F T+Q
$$

these two terms are refined during the correction phase by means of the observed data already predicted by the RT method. So, the Kalman gain is calculated as (12):

$$
G(t)=P(t / t-1) H T(H P(t / t-1) H T+R)-1
$$

the updated equations are given by the following expressions.

$$
\begin{aligned}
& x(t / t)=x(t / t-1)+(z(t)-x(t / t-1) H) G(t) \\
& P(t / t-1)=(I-G(t) H) P(t / t-1))
\end{aligned}
$$

The resulting a-priori and a-posteriori state estimates, and the covariance estimates are saved in the backward step to be used in the smoothing phase. The optimal smoothed estimate of the state is obtained using the observations. In more detail, during the backward step, the state estimate of the forward computed before is updated in order to provide an improved smoothed estimate. The recursive equations are defined as (15):

$$
x(t / n)=x(t / t)-K_{t}(x(t+1 / n)-x(t+1 / t))
$$




$$
P(t / n)=P(t / t)+K_{t}(P(t+1 / n)-P(t+1 / n)) K_{t}^{T}
$$

where $\mathrm{K}_{\mathrm{t}}$ is the smoother gain calculated as,

$$
K_{t}=P(t / t) F^{T}(t+1) P^{-1}(t+1 / t)
$$

the iteration of KS filtering and RTSS is done between adjacent instants in order to ensure the real-time need. In more details, the filtering estimation at time $\mathrm{t}$ is used to smooth the estimated state and covariance at time $\mathrm{t}-1$. This yield to more refined result at time $\mathrm{k}-1$.

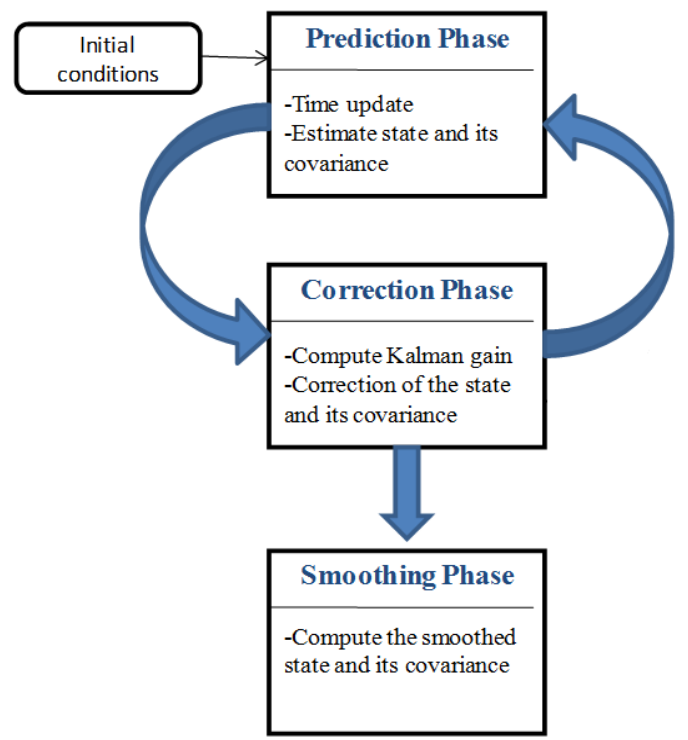

Figure 5. Block diagram of Kalman smoother algorithm

\section{RESULTS AND DISCUSSION}

The proposed algorithm is assessed using real measurements gathered in an indoor environment with size $X=10 \mathrm{~m}$ and $Y=8 \mathrm{~m}$, where 8 reference nodes are deployed at fixed positions. The WSN uses the frequency $2.4 \mathrm{GHz}$. The experimental environment is depicted in Figure 1. For the training data, we consider $\mathrm{M}=285$ training samples uniformly distributed and generated by the pathloss model. The basic metric to evaluate the adopted solution is the relative average localization error $\rho$ defined as (18):

$$
\rho=\frac{1}{N}\left(\sum_{n=1}^{N} \frac{\varepsilon_{n}}{\sqrt{X^{2}+Y^{2}}}\right) \times 100
$$

where $\varepsilon_{n}$ is the absolute error corresponding to position with index $n$.

First, we start looking at the absolute localization error for several test positions. Table 1 shows the predicted positions for the mobile sensor when using KS and KF. The estimation is evaluated for $\mathrm{K}=9$ acquisitions at different time instant. As it can be seen, more accurate position estimation when using KS compared with KF method. For example, the minimum error is $\varepsilon_{k}=0.1 \mathrm{~m}$ at $t=8$ while the maximum value is $\varepsilon_{k}=1.08 \mathrm{~m}$.

Table 1. Absolute error of KS and KF for different time instant

\begin{tabular}{lccccccccc}
\hline time instant $\mathrm{t}$ & $\mathrm{t}=1$ & $\mathrm{t}=2$ & $\mathrm{t}=3$ & $\mathrm{t}=4$ & $\mathrm{t}=5$ & $\mathrm{t}=6$ & $\mathrm{t}=7$ & $\mathrm{t}=8$ & $\mathrm{t}=9$ \\
\hline$\varepsilon[\mathrm{m}]$ for KF & 0.46 & 0.61 & 1.04 & 0.91 & 1.10 & 1.30 & 0.95 & 0.11 & 0.75 \\
$\varepsilon[\mathrm{m}]$ for KS & 0.34 & 0.87 & 0.35 & 0.30 & 0.92 & 1.08 & 0.44 & 0.10 & 0.75 \\
\hline
\end{tabular}

To have fair comparison with the existing state of the art methods, we assess the algorithms in terms of relative localization error and variance. Figure 6 and Figure 7 show the comparison between the proposed 
algorithm, the RT and using KF by focusing on the two metrics. As clearly shown, we obtain lower error and variance with the suggested method. The combination of RT with KS presents an error 5.8\%, while it's $10.6 \%$ and $12.5 \%$ for RT combined to KF and RT respectively. Similarly, the variance of the proposed algorithm is $0.12 \mathrm{~m}$ which is a low value. This proves that our method provides high accuracy for all the estimated positions despite the rapid changes and incertainty that characterize the environement.

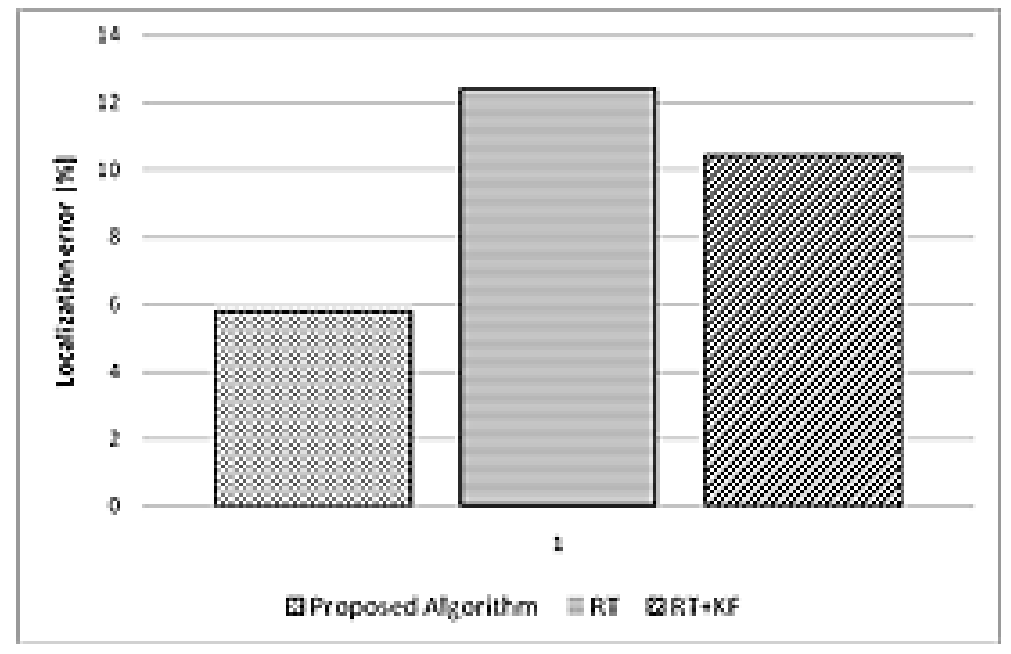

Figure 6. Localization error for the proposed algorithm versus RT, RT+KF

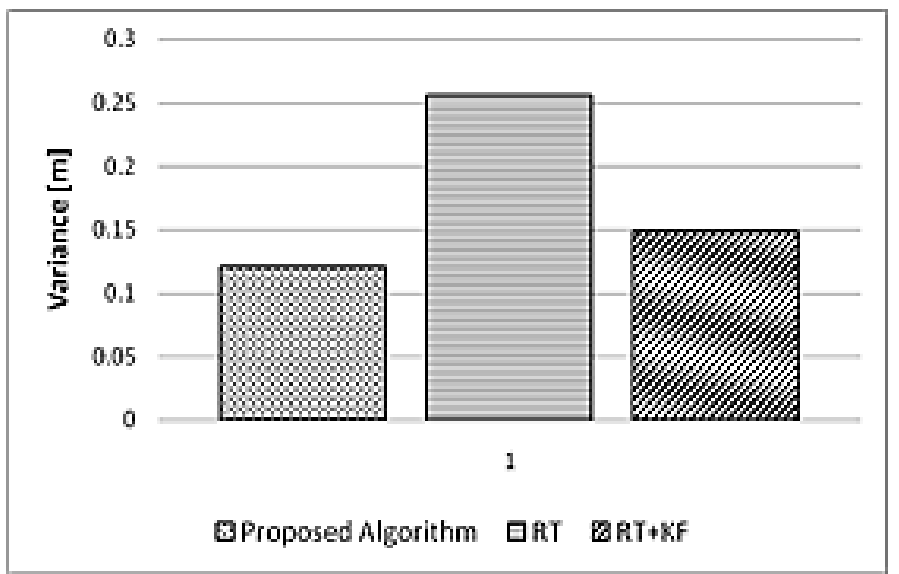

Figure 7. Variance for the proposed algorithm versus RT, RT+KF

Now, we evaluate the impact of the training samples number considerd during the training phase. Consequently, we will vary the distance $\delta$ that separates two training samples as the uniform distribution is considered in this work. Figure 8 and Figure 9 report the average absolute error $\varepsilon$ and its variance in function of the training input data by comparing the proposed algorithm to the existing state of the art strategies: SVR, Naive Bayes, and the trilateration.

In Figures 8 and 9, the suggested method outperforms the other algorithms in terms of error and variance when changing the training samples number. Increasing the number of training samples does not improve the precision. This is due to fluctuations and incertainty of RSSI data. Accordingly, selecting few numbers $\mathrm{M}=285$ training samples will suffice to have optimal results in terms of accuracy and complexity. The comparison among the proposed method and the machine-based localization algorithms: RT, SVR and NB is depicted in Figure 10. As it can be noticed, KS with RT improves the accuracy and outperforms the well known machine learning methods when considering the relative localization error. 


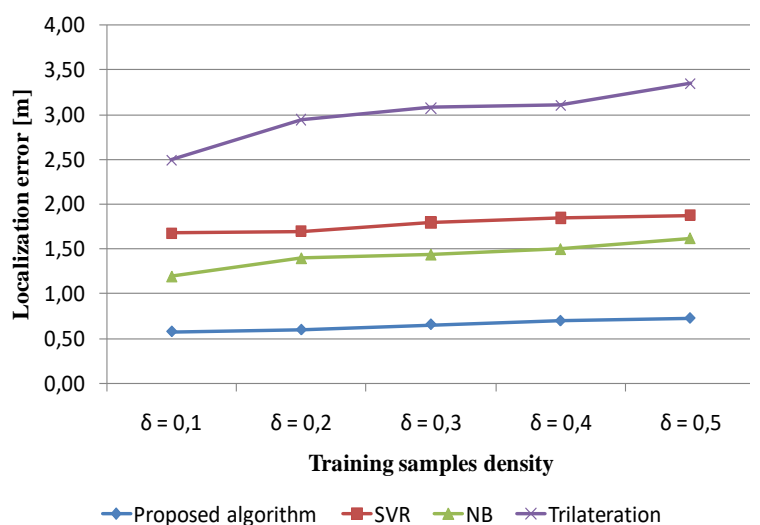

Figure 8. Localization error vs training samples number

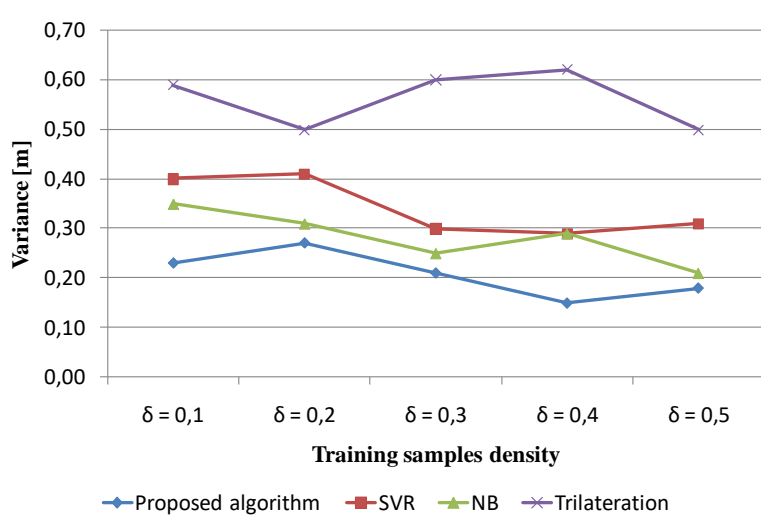

Figure 9. Variance vs training set density

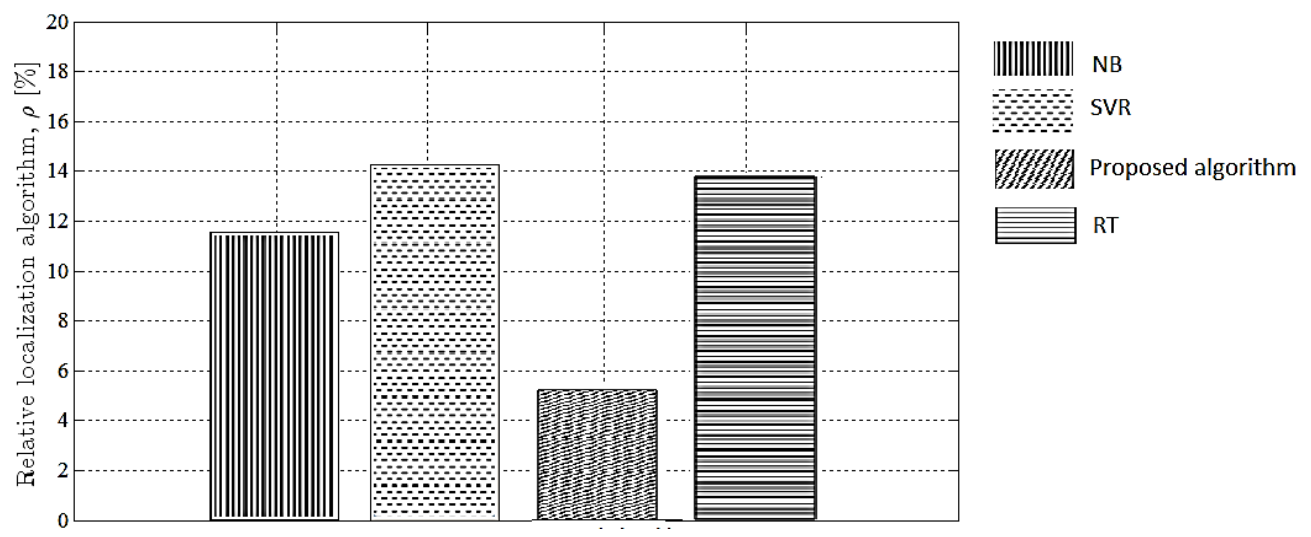

Figure 10. Localization error for the proposed algorithm versus RT, SVR, and NB

\section{CONCLUSION}

In this paper, we address the wireless sensor tracking in an indoor area. The work focuses on using RSSI parameter to reach the accuracy objective. Moreover, this metric is low cost since it'a already available in all wireless equipments. However, this acquired data is submitted to many environmental constraints like fluctuations and incertainty. Consequently, we propose to use machine learning algorithm to overcome the issue of the environment complexity. During the training phase, the algorithm starts the data collection by distributing the training samples in the area and determining the corresponding RSSI using the pathloss model. During the testing phase, real RSSI measurements of a mobile sensor are considered as the input of the trained model. Consequently, the positions are estimated using the regression tree model already built during the training step. As our major concern is accurate positionning, the regression tree is combined with the Kalman Smoother method yielding to more refined localization estimation. The suggested method has been evaluated using real RSSI measurements and compared to existing learning by example algorithms. The results show the suggested approach outperforms other methods, giving low localization error.

As mentioned before, the RSSI presents low-cost metric, while this radio parameter is too sensitive particularly when considering dynamic scenario in an indoor environment. Accordingly, efficient techniques of filtering would be studied and discussed to reduce the real RSSI measurements fluctuations together with the improvement of the propagtion model. Moreover, real time availability is one of the localization goals that would improve. The delay of the position estimation would be reduced particularly when dealing with emergency applications.

Future work intends to improve the suggested approach by considering multi moving targets. Also, the method will be adapted to a more complex indoor environment with obstacles. On the other side, the sensor node is limited in terms of battery. Reducing the computational cost of the localization method is a major concern. Thus, the next challenge is to reduce the algorithm complexity. 


\section{REFERENCES}

[1] T. Wu, F. Wu, C. Qiu, J.-M. Redouté, and M. R. Yuce, "A Rigid-Flex Wearable Health Monitoring Sensor Patch for IoTConnected Healthcare Applications," in IEEE Internet of Things Journal, vol. 7, no. 8, pp. 6932-6945, Aug. 2020, doi: 10.1109/JIOT.2020.2977164.

[2] Md. W. Rahman, Md. E. Hossain, R. Islam, Md. H. A. Rashid, Md. N. A. Alam, and Md. M. Hasan, "Real-time and Low-cost IoT based farming using raspberry Pi," Indonesian Journal of Electrical Engineering and Computer Science, vol. 17, no. 1, pp. 197204, 2020, doi: 10.11591/ijeecs.v17.i1.pp197-204.

[3] H. Nawaz, A. Bozkurt, and I. Tekin, "A novel power efficient asynchronous time difference of arrival indoor localization system using CC1101 radio transceivers," Microwave and Optical Technology Letters, vol. 59, no. 3, pp. 550-555, 2017, doi: 10.1002/mop.30342.

[4] R. M. Dellosa, A. C. Fajardo, and R. P. Medina, "Modified fingerprinting localization technique of indoor positioning system based on coordinates," Indonesian Journal of Electrical Engineering and Computer Science, vol. 15, no. 3, pp. 1345-1355, 2019, doi: 10.11591/ijeecs.v15.i3.pp1345-1355.

[5] V. Bianchi, P. Ciampolini, and I. De Munari, "RSSI-Based Indoor Localization and Identification for ZigBee Wireless Sensor Networks in Smart Homes," in IEEE Transactions on Instrumentation and Measurement, vol. 68, no. 2, pp. 566-575, Feb. 2019, doi: 10.1109/TIM.2018.2851675.

[6] M. T. Garip, P. H. Kim, P. Reiher, and M. Gerla, "INTERLOC: An interference-aware RSSI-based localization and sybil attack detection mechanism for vehicular ad hoc networks," 2017 14th IEEE Annual Consumer Communications \& Networking Conference (CCNC), 2017, pp. 1-6, doi: 10.1109/CCNC.2017.8013424.

[7] M. Vaquar and S. K. Agarwal, "Target Object Tracking with Portable Sensors in Wireless Sensor Network," in International Journal of Engineering and Advanced Technology (IJEAT), vol. 9, no. 2, pp. 2505-2510, 2019. doi: 10.1109/JSEN.2017.2660522.

[8] F. Wen and C. Liang, "Fine-Grained Indoor Localization Using Single Access Point with Multiple Antennas," in IEEE Sensors Journal, vol. 15, no. 3, pp. 1538-1544, March 2015, doi: 10.1109/JSEN.2014.2364121.

[9] S. Wu, S. Zhang, K. Xu, and D. Huang, "Neural Network Localization with TOA Measurements Based on Error Learning and Matching," in IEEE Access, vol. 7, pp. 19089-19099, 2019, doi: 10.1109/ACCESS.2019.2897153.

[10] Y. Jin, W.-S. Soh, and W.-C. Wong, "Indoor localization with channel impulse response-based fingerprint and nonparametric regression," in IEEE Transactions on Wireless Communications, vol. 9, no. 3, pp. 1120-1127, March 2010, doi: 10.1109/TWC.2010.03.090197.

[11] F. Zhu and J. Wei, "Localization Algorithm for Large Scale Wireless Sensor Networks Based on Fast-SVM," Wireless Personal Communications: An International Journal, vol. 95, no. 3, pp. 1859-1875, 2017, doi: 10.1007/s11277-016-3665-2.

[12] S. Amri, F. Khelifi, A. Bradai, A. Rachedi, M. L. Kaddachi, and M. Atri, "A new fuzzy logic-based node localization mechanism for Wireless Sensor Networks," Future Generation Computer Systems, vol. 93, pp. 799-813, 2019, doi: 10.1016/j.future.2017.10.023.

[13] I. Ullah, Y. Shen, X. Su, C. Esposito, and C. Choi, "A Localization Based on Unscented Kalman Filter and Particle Filter Localization Algorithms," in IEEE Access, vol. 8, pp. 2233-2246, 2020, doi: 10.1109/ACCESS.2019.2961740.

[14] L. Li, W. Xie, and Z. Liu, “Auxiliary truncated particle filtering with least-square method for bearings-only maneuvering target tracking," in IEEE Transactions on Aerospace and Electronic Systems, vol. 52, no. 5, pp. 2562-2567, October 2016, doi: 10.1109/TAES.2016.150048.

[15] M. Lipka, E. Sippel, and M. Vossiek, “An Extended Kalman Filter for Direct, Real-Time, Phase-Based High Precision Indoor Localization," in IEEE Access, vol. 7, pp. 25288-25297, 2019, doi: 10.1109/ACCESS.2019.2900799.

[16] F. T. AL-Dhief et al., "Performance comparison between TCP and udp protocols in different simulation scenarios," International Journal of Engineering \& Technology, vol. 7, no. 4, pp. 172-176, 2018, doi: 10.14419/ijet.v7i4.36.23739.

[17] R. Duvignau, B. Havers, V. Gulisano, and M. Papatriantafilou, "Time- and Computation-Efficient Data Localization at Vehicular Networks' Edge," in IEEE Access, vol. 9, pp. 137714-137732, 2021, doi: 10.1109/ACCESS.2021.3118596.

[18] A. A. Ababneh, "Knapsack-Based Sensor Selection for Target Localization Under Energy and Error Constraints," in IEEE Sensors Journal, vol. 21, no. 23, pp. 27208-27217, 1 Dec.1, 2021, doi: 10.1109/JSEN.2021.3123734.

[19] F. Affane, K. Z. Meguenni, and A. Omari, "Type-2 fuzzy logic controller optimized by wavelet networks for mobile robot navigation," Indonesian Journal of Electrical Engineering and Computer Science (IJEECS), vol. 18, no. 1, pp. 326-334, 2020, doi: 10.11591/ijeecs.v18.i1.pp326-334.

[20] X. Liu et al., "Accurate Localization of Tagged Objects Using Mobile RFID-Augmented Robots," in IEEE Transactions on Mobile Computing, vol. 20, no. 4, pp. 1273-1284, 1 April 2021, doi: 10.1109/TMC.2019.2962129.

[21] S. H. Abdulredah and D. J. Kadhim, "Developing a real time navigation for the mobile robots at unknown environments," Indonesian Journal of Electrical Engineering and Computer Science, vol. 20, no. 1, pp. 500-509, 2020, doi: 10.11591/ijeecs.v20.i1.pp500-509.

[22] F. Zhao, R. Xu, R. Li, M. Zhu, and X. Luo, "Street-Level Geolocation Based on Router Multilevel Partitioning," in IEEE Access, vol. 7, pp. 59237-59248, 2019, doi: 10.1109/ACCESS.2019.2914972.

[23] P. Singh, R. S. Raw, S. A. Khan, M. A. Mohammed, A. A. Aly, and D. -N. Le, "W-GeoR: Weighted Geographical Routing for VANET's Health Monitoring Applications in Urban Traffic Networks," in IEEE Access, doi: 10.1109/ACCESS.2021.3092426.

[24] S. A. Mostafa et al., "An agent architecture for autonomous UAV flight control in object classification and recognition missions," Soft Computing, 2021, doi: 10.1007/s00500-021-05613-8.

[25] A. Eskandarian, C. Wu, and C. Sun, "Research Advances and Challenges of Autonomous and Connected Ground Vehicles," in IEEE Transactions on Intelligent Transportation Systems, vol. 22, no. 2, pp. 683-711, Feb. 2021, doi: 10.1109/TITS.2019.2958352.

[26] Z. Zhao, L. Zhou, and Q. Zhu, "Preview distance adaptive optimization for the path tracking control of unmanned vehicle," Journal of Mechanical Engineering, vol. 54, no. 24, pp. 166-173, 2018, doi: 10.3901/JME.2018.24.166.

[27] H. Marzbani, H. Khayyam, C. N. TO, Đ. V. Quoc, and R. N. Jazar, "Autonomous Vehicles: Autodriver Algorithm and Vehicle Dynamics," in IEEE Transactions on Vehicular Technology, vol. 68, no. 4, pp. 3201-3211, April 2019, doi: 10.1109/TVT.2019.2895297.

[28] S. Xu and H. Peng, "Design, Analysis, and Experiments of Preview Path Tracking Control for Autonomous Vehicles," in IEEE Transactions on Intelligent Transportation Systems, vol. 21, no. 1, pp. 48-58, Jan. 2020, doi: 10.1109/TITS.2019.2892926.

[29] R. P. D. Vivacqua, M. Bertozzi, P. Cerri, F. N. Martins, and R. F. Vassallo, "Self-Localization Based on Visual Lane Marking Maps: An Accurate Low-Cost Approach for Autonomous Driving," in IEEE Transactions on Intelligent Transportation Systems, vol. 19, no. 2, pp. 582-597, Feb. 2018, doi: 10.1109/TITS.2017.2752461. 
[30] S. H. Thimmaiah and G. Mahadevan, "A Range Based Localization Error Minimization Technique for Wireless Sensor Network," Indonesian Journal of Electrical Engineering and Computer Science, vol. 7, no. 2, pp. 395-403, 2017, doi: 10.11591/ijeecs.v7.i2.pp395-403.

[31] S. S. Dias and M. G. S. Bruno, "Cooperative Target Tracking Using Decentralized Particle Filtering and RSS Sensors," in IEEE Transactions on Signal Processing, vol. 61, no. 14, pp. 3632-3646, July15, 2013, doi: 10.1109/TSP.2013.2262276.

[32] C. Perez-Vega and J. L. G. Garcia, "A Simple Approach to a Statistical Path Loss Model for Indoor Communications," 1997 27th European Microwave Conference, 1997, pp. 617-623, doi: 10.1109/EUMA.1997.337753.

[33] H. Ahmadi, F. Viani, A. Polo, and R. Bouallegue, "Learning ensemble strategy for static and dynamic localization in wireless sensor networks," International Journal of Network Management, vol. 27, no. 4, 2017, doi: 10.1002/nem.1979.

\section{BIOGRAPHIES OF AUTHORS}
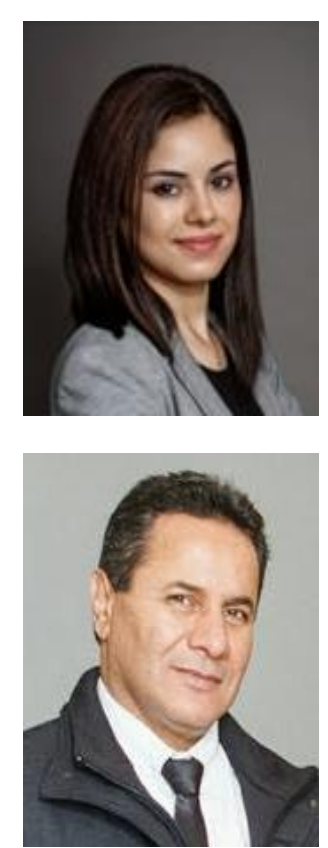

Hanen Ahmadi (iD SS SC P received the Engineering, research master and $\mathrm{PhD}$ degrees in communication systems from the National Engineering School of Tunis (ENIT), El Manar University, in 2011, 2012 and 2017, respectively. She is now an assistant professor and researcher at Innov'COM Laboratory at SupCom in Tunisia. Her research areas include the Localization in Wireless Sensor Networks, Wireless Networks, M2M and signal processing. She can be contacted at email: hanen.ahmadi@ medtech.tn.

Ridha Bouallegue (D) SC $\mathrm{P}$ is professor at the National Engineering School of Tunis, Tunisia (ENIT) with experience in teaching since 1990. Since 1995, he is professor at the High School of Communications of Tunis (Sup'Com). He obtained his doctorate in 1998 and his HDR in 2003 on the multi-user detection in cellular radio systems of the next generation. His research and fundamental development are on the physical layer of communication systems, particularly on digital communications systems and information theory, the next generation of wireless networks, and Wireless Communications. He can be contacted at email: ridha.bouallegue@supcom.tn. 